\title{
Non-pharmacological Interventions in Anorexia of Aging
}

\author{
J.G. Ruiz ${ }^{1,2}$ \\ 1. Miami VA Healthcare System, Geriatric Research, Education and Clinical Center (GRECC), Florida, USA; 2. University of Miami Miller School of Medicine, Florida, USA
}

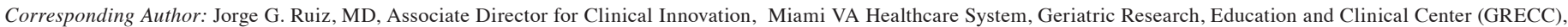
Florida, USA, Electronic mail: j.ruiz@med.miami.edu

A norexia of aging -a common geriatric syndrome- is defined as appetite loss, reduced food intake or both in older adults (1). Anorexia of aging is associated with functional impairment, frailty, institutionalization, weight loss, and when leading to malnutrition, all-cause mortality (2-4). The pathophysiology of this syndrome is complex involving the interplay of central and peripheral hormones and neurotransmitters; age-related impairments in the sense of smell and taste; and inflammation $(1,5)$. Research has revealed multiple risk factors including prescription of anorexigenic drugs, multimorbidity, disability, masticatory impairment, neurocognitive disorders, depression, socio-cultural issues and ageist attitudes $(1,6)$. The assessment consists of a complete history and physical, use of validated instruments (e.g., short nutritional assessment questionnaire, SNAQ), evaluation of nutritional status (e.g., mini-nutritional assessment, MNA) and judicious laboratory testing (7). As in other geriatric syndromes, multicomponent interventions are most likely to be effective $(4,8)$. The management of anorexia of aging targets possible causes and encompasses pharmacological and non-pharmacological interventions. Despite efforts by the pharmaceutical industry, neither the US Food and Drug Administration (FDA) nor the European Medicine Agency (EMA) have approved orexigenic drugs for this indication. Non-pharmacological interventions represent common sense, yet available, and potentially effective and safe approaches to improve older persons' appetite and prevent malnutrition and its multiple sequelae.

Research into the benefits of non-pharmacological interventions to treat anorexia of aging remains disappointingly small (9). Besides evaluating specific strategies, an issue to consider is the timing of the non-pharmacological interventions. The preventive stages represent a useful framework for investigating efficacy and effectiveness: risk for anorexia (primary prevention); anorexia alone (secondary prevention); and anorexia associated with weight loss or malnutrition (tertiary prevention). Within this conceptualization, nonpharmacological interventions are considered first-line strategies to prevent and treat anorexia of aging. Nonpharmacological interventions consist of various strategies that may fall into diverse domains $(1,9)$. In terms of primary prevention, clinicians in primary care settings may implement educational interventions including nutritional counseling; address the ageist attitudes of both patients, caregivers, and clinicians towards age-related appetite loss; and avoid or deprescribe anorexigenic drugs. Meal adjustments may require careful attention to food palatability, presentation, variety, and odor. Providing culturally appropriate, affordable meals would ensure older persons' adherence and satisfaction. Social-environmental approaches include socialization; feeding assistance for older individuals with functional or cognitive impairment; and supplementary meal assistance (e.g., meals on wheels programs). Food insecurity -the inadequate access to healthy food- is a critical social determinant to consider when designing non-pharmacological interventions $(10,11)$. Exercise and physical activity stimulates appetite while offering other health benefits. In addition to the aforementioned interventions, secondary prevention may involve dietitian-directed counseling as an integral part of an interprofessional geriatrics team. Tertiary prevention includes a range of nutritional interventions including the use of oral caloric-protein supplements and fortified foods in addition to an intensification of the primary and secondary prevention strategies. At each level of prevention, the role of caregivers is key as they are often the first to recognize anorexia and participate in the implementation of an individualized treatment plan.

The JNHA welcomes submissions of observational and experimental studies that considering the proposed preventive care framework, report on the efficacy and effectiveness of non-pharmacological interventions. In addition to improving appetite, other outcomes to consider are weight loss, malnutrition, functional status, sarcopenia, frailty and healthcare utilization. Studies reporting person-centered outcomes, quality of life measures and caregiver feedback are essential. Research investigating the role of caregivers, dietitians and interprofessional geriatric teams; cultural issues, health disparities and inequality; and ethical issues are other important research areas. The emergence of the COVID 19 pandemic, which has affected mostly older populations, calls for studies that examine the potential effects of postCOVID conditions on appetite. Another area is research that examines the combination of non-pharmacological approaches with orexigenic agents. Drugs may still have a role, but it is unclear whether the combination with non-pharmacological measures would be indicated during the secondary or rather, later in the tertiary prevention stage. All the proposed studies should include vulnerable older adults in settings that span the spectrum of geriatric care -community, hospital, long-term care, and palliative care and hospice.

Hence, lacking effective pharmacological treatments lends 
an urgency to investigate non-pharmacological interventions to prevent, or treat anorexia of aging. In addition to their potential clinical benefits, non-pharmacological interventions are consistent with an ethos of geriatric care that fosters older adults' dignity, autonomy, and quality of life.

Conflicts of interest: Pfizer (Consultant).

\section{References}

1. Landi, F., et al., Anorexia of Aging: Risk Factors, Consequences, and Potential Treatments. Nutrients, 2016. 8(2): p. 69. DOI: 10.3390/nu8020069

2. Landi, F., et al., Anorexia, physical function, and incident disability among the frail elderly population: results from the ilSIRENTE study. J Am Med Dir Assoc, 2010 11(4): p. 268-74. DOI: 10.1016/j.jamda.2009.12.088

3. Roy, M., P. Gaudreau, and H. Payette, A scoping review of anorexia of aging correlates and their relevance to population health interventions. Appetite, 2016. 105: p. 688-99. DOI: 10.1016/j.appet.2016.06.037
4. Merchant, R.A., J. Woo, and J.E. Morley, Editorial: Anorexia of Ageing: Pathway to Frailty and Sarcopenia. J Nutr Health Aging, 2022. 26(1): p. 3-5. DOI: $10.1007 /$ s12603-021-1703-6

5. Morley, J.E., Pathophysiology of the anorexia of aging. Curr Opin Clin Nutr Metab Care, 2013. 16(1): p. 27-32. DOI: 10.1097/MCO.0b013e328359efd7

6. Morley, J.E., Anorexia of aging: a true geriatric syndrome. J Nutr Health Aging, 2012. 16(5): p. 422-5. DOI: 10.1007/s12603-012-0061-9

7. Jadczak, A.D. and R. Visvanathan, Anorexia of Aging - An Updated Short Review. J Nutr Health Aging, 2019. 23(3): p. 306-309. DOI: 10.1007/s12603-019-1159-0

8. Cox, N.J., et al., New horizons in appetite and the anorexia of ageing. Age Ageing, 2020. 49(4): p. 526-534. DOI: 10.1093/ageing/afaa014

9. Cox, N.J., et al., Assessment and Treatment of the Anorexia of Aging: A Systematic Review. Nutrients, 2019. 11(1). DOI: 10.3390/nu11010144

10. Coleman-Jensen, A., et al., Household food security in the United States in 2016-A report summary from the Economic Research Service of the United States Department of Agriculture. September 2017. Retrieved June, 2018. 11.

11. Pooler, J.A., et al., Food Insecurity: A Key Social Determinant of Health for Older Adults. J Am Geriatr Soc, 2019. 67(3): p. 421-424. DOI: 10.1111/jgs.15736

How to cite this article: J.G. Ruiz. Non-pharmacological Interventions in Anorexia of Aging. J Nutr Health Aging. 2022;26(2):110-111, http://dx.doi.org/10.1007/s12603-022$1744-5$ 